\title{
Highly Oriented Nanowires From the Hierarchical Self-Assembly in Supramolecular Complex of Polyaniline with $\omega$-Methoxy Poly(ethylene oxide) Phosphates
}

\author{
Bhanu Nandan, ${ }^{1}$ Jen-Yung Hsu, ${ }^{1}$ Ayano Chiba, ${ }^{2}$ Hsin-Lung Chen, ${ }^{1} *$ Chein-Shiun \\ Liao, ${ }^{3}$ Show-An Chen, ${ }^{1}$ Hirokazu Hasegawa ${ }^{2}$ \\ ${ }^{1}$ Department of Chemical Engineering, National Tsing Hua University, Hsin-Chu \\ 30013, Taiwan; ${ }^{2}$ Department of Polymer Chemistry, Graduate School of \\ Engineering, Kyoto University, Nishikyo-ku, Kyoto 616-8510, Japan; \\ ${ }^{3}$ Department of Chemical Engineering and Material Science, Yuan Ze University, \\ Nei-Li, Taoyuan 320, Taiwan
}

\section{$\underline{\text { Supporting Information }}$}

\section{Synthetic Procedures}

Synthesis of PEOPA. The PEOPA was synthesized according to a general procedure for synthesis of phosphate derivatives by reaction of phosphorus pentaoxide and hydroxyl compound (Polymer 1999, 40, 5723) as shown in Scheme 1. The limiting reagent of poly(ethylene glycol) monmethyl ether $\left(\mathrm{CH}_{3}\left(\mathrm{OCH}_{2} \mathrm{CH}_{2}\right)_{\mathrm{n}} \mathrm{OH}\right)$ (PEGME) $\left(\mathrm{M}_{\mathrm{n}}=550\right)$ was reacted with $\mathrm{P}_{2} \mathrm{O}_{5}$ by a mole ratio of 3:1 which led to a stoichiometric mixture of mono- and bi-hydroxy acid with molar ratio of 1:1 due to the much larger $K_{\mathrm{a} 1}\left(7.1 \times 10^{-3}\right)$ of phosphorus acid than $K_{\mathrm{a} 2}$ $\left(6.3 \times 10^{-8}\right)$. In a typical reaction, $0.3 \mathrm{~mol}$ of PEGME in $50 \mathrm{ml}$ benzene was added into a dispersion of 0.1 mol $\mathrm{P}_{2} \mathrm{O}_{5}(14.16 \mathrm{~g})$ in $50 \mathrm{ml}$ benzene. The mixture was heated to $70{ }^{\circ} \mathrm{C}$ and stirred for 3 hours. After removing residual $\mathrm{P}_{2} \mathrm{O}_{5}$ and solvent, a colorless transparent liquid was obtained. As shown in Scheme 1 the product (I and II) is a mixture of mono- and bi-hydroxyl acids with a molar ratio of 1:1. The average molecular weight of the PEOPA was estimated to 
be 896 according to $1: 1$ mixture of $\left(\mathrm{CH}_{3}\left(\mathrm{OCH}_{2} \mathrm{CH}_{2}\right)_{\mathrm{n}} \mathrm{OPO}(\mathrm{OH})_{2}\right.$ and $\left.\left[\mathrm{CH}_{3}\left(\mathrm{OCH}_{2} \mathrm{CH}_{2}\right)_{\mathrm{n}}\right]_{2} \mathrm{OPO}(\mathrm{OH})\right)$.

The molecular weight of PEOPA was characterized by mass spetrum (FAB mode). There were two $\mathrm{m} / \mathrm{z}(\mathrm{MH})^{-} 1139$ and 1095 observed in the MS spectrum in Figure 1S. The PEGME used for the PEOPA synthesis was a mixture of PEO oligomer with the number of repeating unit $n$ of $11 \sim 12$. The $m / z(\mathrm{MH})^{-} 1139$ corresponds to the PEOPA containing two PEO chains (i.e., disubstituted PEOPA) with one having $n=11$ and the other having $n=12$. The $m / z(\mathrm{MH})^{-} 1095$ signal is associated with the PEOPA containing two PEO chains with $n=11$. The (MH) ${ }^{-}$peaks at $m / z 597$ and 641 correspond to PEOPA with mono-substituted PEO chain with $n=11$ and $n=$ 12, respectively. The elemental analysis calculated for PEOPA with 1:1 mixture of di- and mono-substituted PEO chains with $n=11$ is $\mathrm{C} 48.99 \%$ and $\mathrm{H} 8.52 \%$. The element analysis found is $\mathrm{C} 49.87 \%$ and $\mathrm{H} 9.62 \%$, which closely agree with the calculated values The chemical shift of ${ }^{1} \mathrm{H}$ NMR for the methylene protons in PEOPA appears at $\delta=3.60-3.61 \mathrm{ppm}$; the methoxy protons at the chain end of PEOPA appears at $\delta=3.33 \mathrm{ppm}$. The peaks for the two hydroxyl protons of phosphate appear in two groups at $\delta=4.10-4.13 \mathrm{ppm}$ and $\delta=4.26-4.28 \mathrm{ppm}$, whose integral ratio indicates a 1:1 mixture of mono- and di-substituted phosphate. 


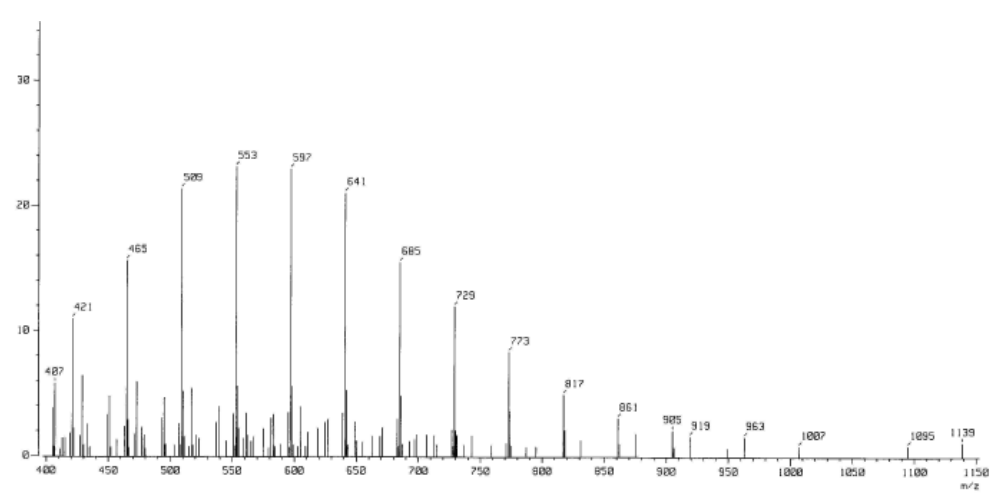

Fig. 1S The mass spectrum of PEOPA (FAB mode).

Synthesis of PANI. PANI was synthesized by the oxidative polymerization of its monomers in $1 \mathrm{M}$ aqueous $\mathrm{HCl}$ with $\left(\mathrm{NH}_{4}\right)_{2} \mathrm{~S}_{2} \mathrm{O}_{8}$ as oxidant followed by further reducing to $\mathrm{EB}$ form (III) with dilute ammonium hydroxide solution according to literature procedures. The molecular weight of PANI was measured by GPC using NMP as the solvent. The result was as followed: $\mathrm{M}_{\mathrm{w}}=65,149 ; \mathrm{M}_{\mathrm{n}}=39,343 ; \mathrm{PDI}=1.66$.

Complex Preparation. PANI(PEOPA) complex with $\mathrm{R}=0.5$ were prepared from aqueous solution, where $\mathrm{R}$ denotes the binding fraction given by the average number of PEOPA molecules bound to one monomer unit of PANI. PANI/PEOPA physical mixture in suitable molar ratio was dissolved in freshly distilled water at a concentration of $20 \mathrm{mg} / \mathrm{mL}$. The aqueous solution was subsequently magnetically stirred for five days at room temperature to obtain a dark solution. The formation of PANI(PEOPA) $)_{R}$ complex (IV) is shown in Scheme 2. The conducting film of the PANI(PEOPA $)_{\mathrm{R}}$ complex was obtained from the preceding solution by casting it in a Petri dish, drying on a hot plate at $50{ }^{\circ} \mathrm{C}$ followed by further drying in vacuo at 40 ${ }^{\circ} \mathrm{C}$ for $24 \mathrm{hrs}$. 


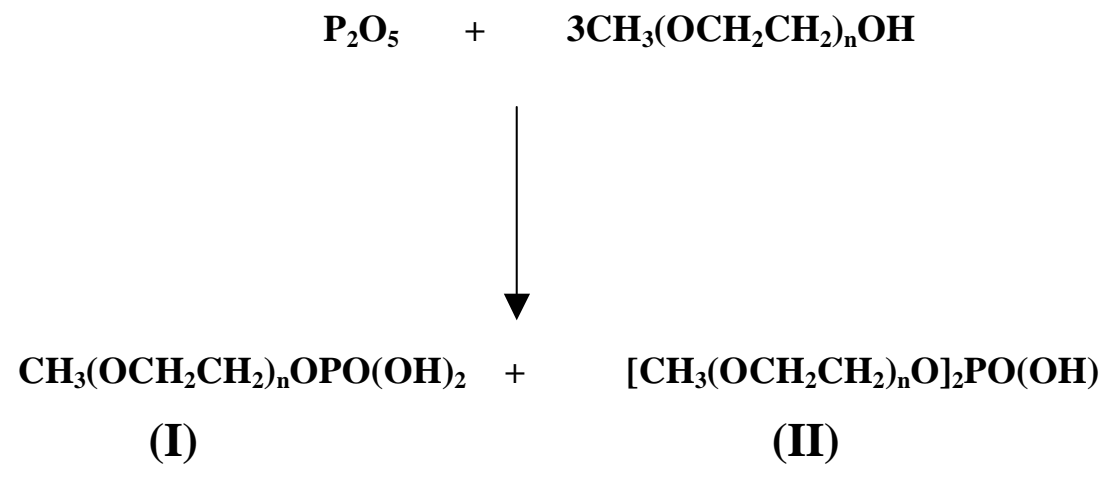

Scheme 1 


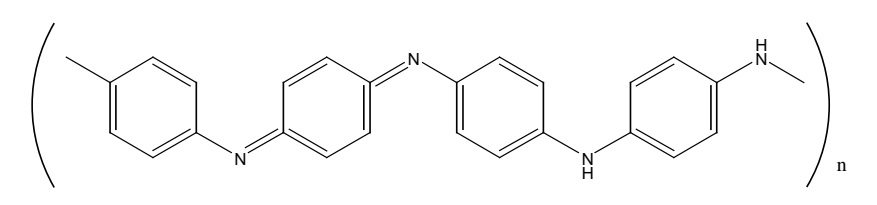

(III)

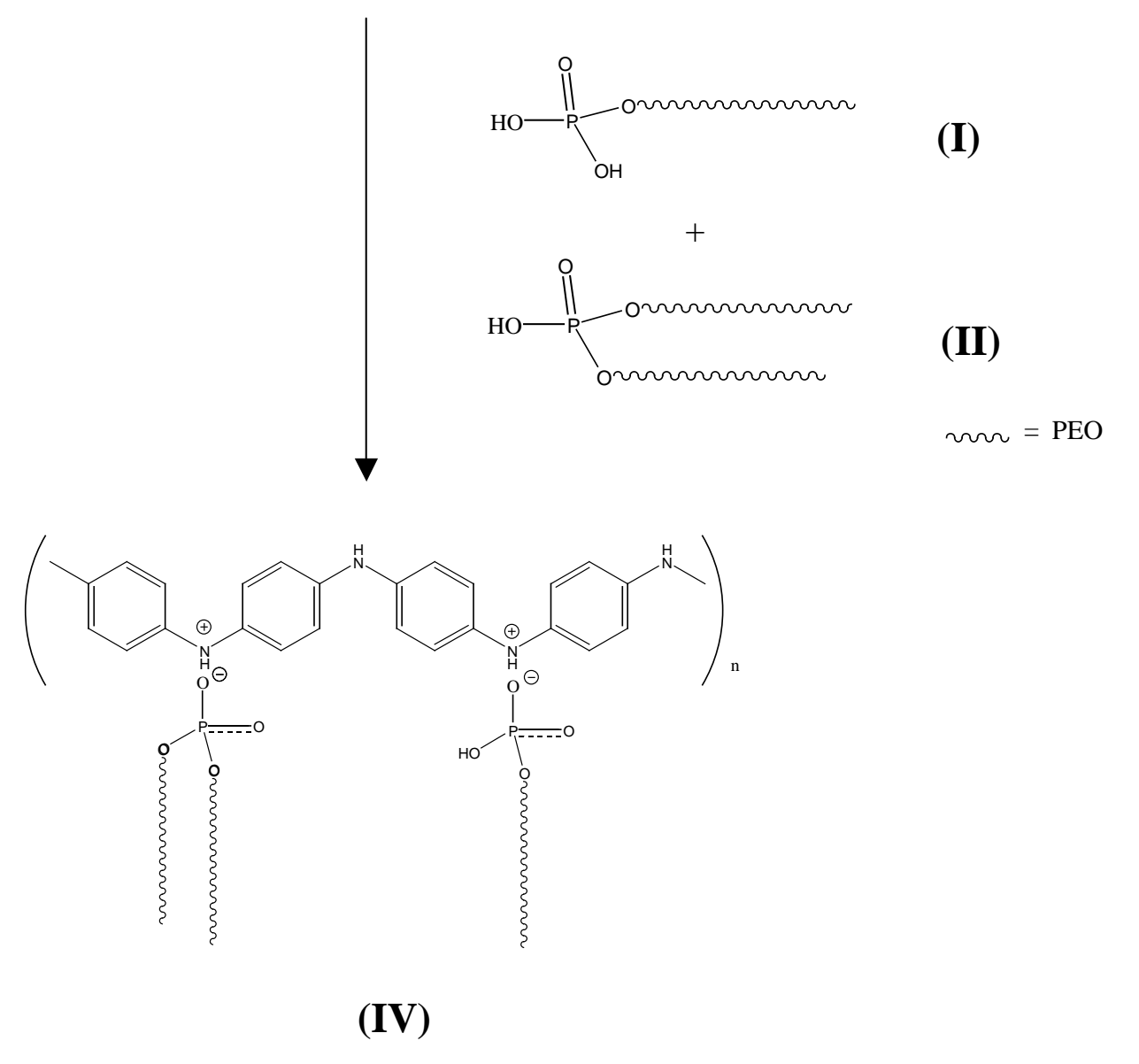

Scheme 2 


\section{Characterization Details}

UV-Vis Spectroscopy. An ultraviolet-visible spectrophotometer (UV-vis spectrometer, Jasco 310) was utilized to verify the complex formation. The spectra in the wavelength range of 200$1200 \mathrm{~nm}$ were recorded from the $1 \mathrm{mg} / \mathrm{mL}$ aqueous solutions of PANI(PEOPA) complexes.

TEM Measurements. The real-space morphology of PANI(PEOPA) complexes was observed by a JEM-2000EX II transmission electron microscope operated at $100 \mathrm{kV}$. The film specimens were microtomed along three mutually perpendicular directions at $-90{ }^{\circ} \mathrm{C}$ using a Reichert Ultracut E low-temperature sectioning system. The ultrathin sections were picked onto the copper grids coated with carbon-supporting films followed by staining by exposure to the vapor of $1 \% \mathrm{OsO}_{4}$ (aq.) for $2-3 \mathrm{~h}$.

2D Synchrotron Small-Angle X-ray Scattering Measurements. The 2D synchrotron SAXS experiments were conducted at BL15-A SAXS beamline at the Photon Factory of High-Energy Accelerator Research Organization (KEK) in Tsukuba, Japan. The scattered beam was recorded using a charge-coupled device $(C C D)$ area detector covering a scattering vector $q=4 \pi / \lambda \sin (\theta / 2)$ $\left(\theta=\right.$ scattering angle) range from 0.1 to $2.0 \mathrm{~nm}^{-1}$. The obtained scattering patterns were corrected for the air scattering as well as nonuniformity, image distortion, and dark current of the detector. 


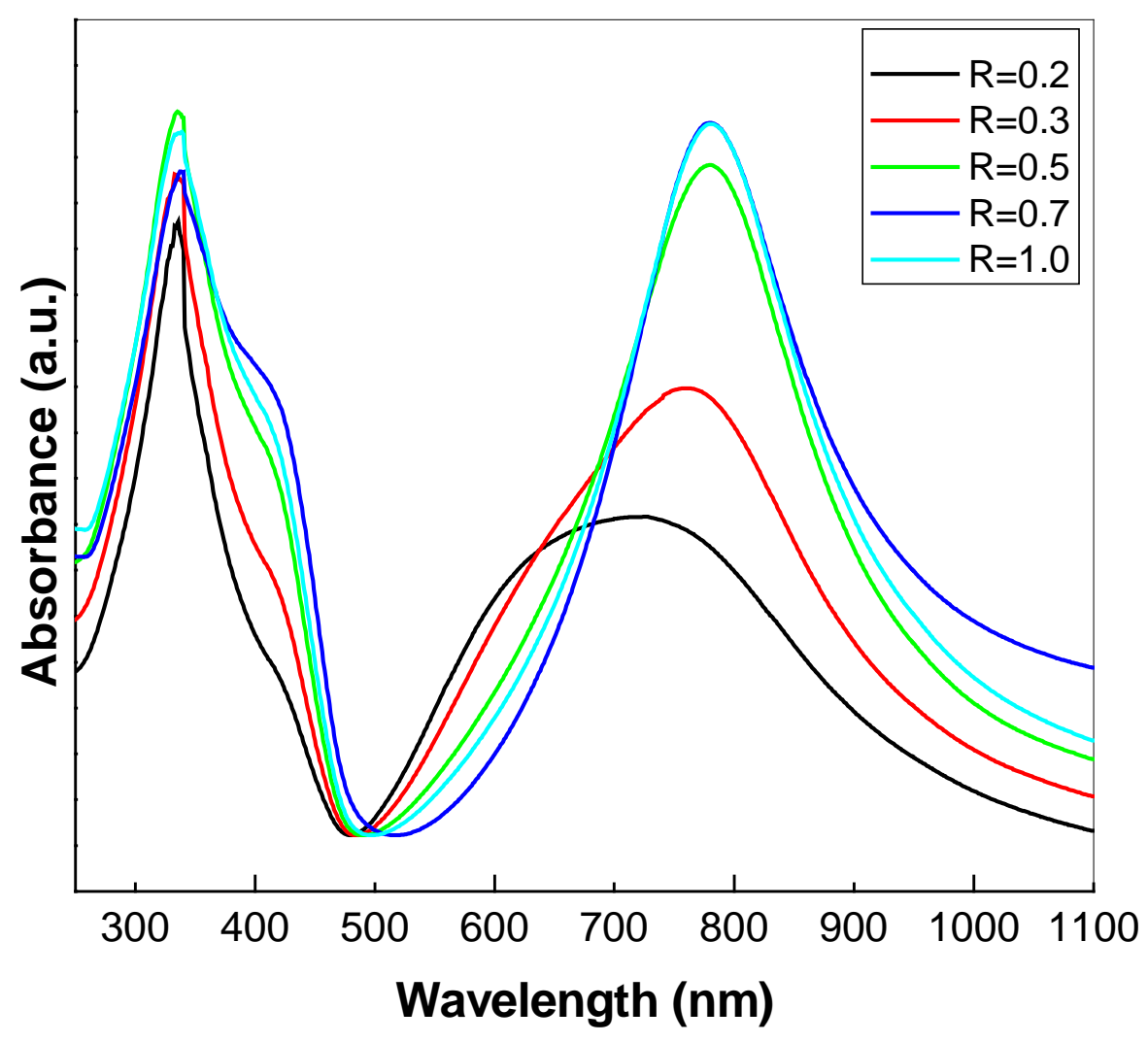

Figure 2S. UV-Vis absorption spectra of PANI(PEOPA $)_{\mathrm{R}}$ complexes with different binding fractions measured in aqueous solution. The commonly reported UV-Vis spectrum of PANI has two absorption peaks near 300 and $600 \mathrm{~nm}$, which has been attributed to the $\pi-\pi^{*}$ transition of the benzenoid rings and the exciton absorption of the quinoid rings, respectively. The PANI structure in the emeraldine base form has half of the nitrogen atoms in amine groups and the other half in imine groups. While the nitrogen atoms in the imine groups are protonated, nitrogen and its neighboring quinoid ring become a semiquinoid radical cation and the intensity of exciton absorption peak near $600 \mathrm{~nm}$ decreases. Hence the intensity of peak near $600 \mathrm{~nm}$ gives an idea about the extent of doping of PANI chains. ${ }^{1}$ As can be observed from Figure 1S, the intensity of the exciton absorption peak decreased as the binding fraction increased indicating increased 
doping at higher binding fraction. At lower binding fractions of $\mathrm{R}=0.2$ and 0.3 , the exciton peak still persists which indicates incomplete doping of PANI chains. Even at $\mathrm{R}=0.5$ the exciton absorption peak persists though with considerably reduced intensity. The doping of PANI chains can also be followed by the appearance of absorption peaks due to polaron band transitions at around 400 and $800 \mathrm{~nm}$. As can be observed from Figure 2, two new peaks emerge near 400 and $800 \mathrm{~nm}$ with their intensities increasing with increasing binding fraction, which reveals a higher extent of doping at larger R. Thus the UV-Vis data gives enough information on the extent of doping of PANI chains by PEOPA.

\section{References}

1. Chen, S. A.; Lee, H.-T. Macromolecules 1995, 28, 2858. 


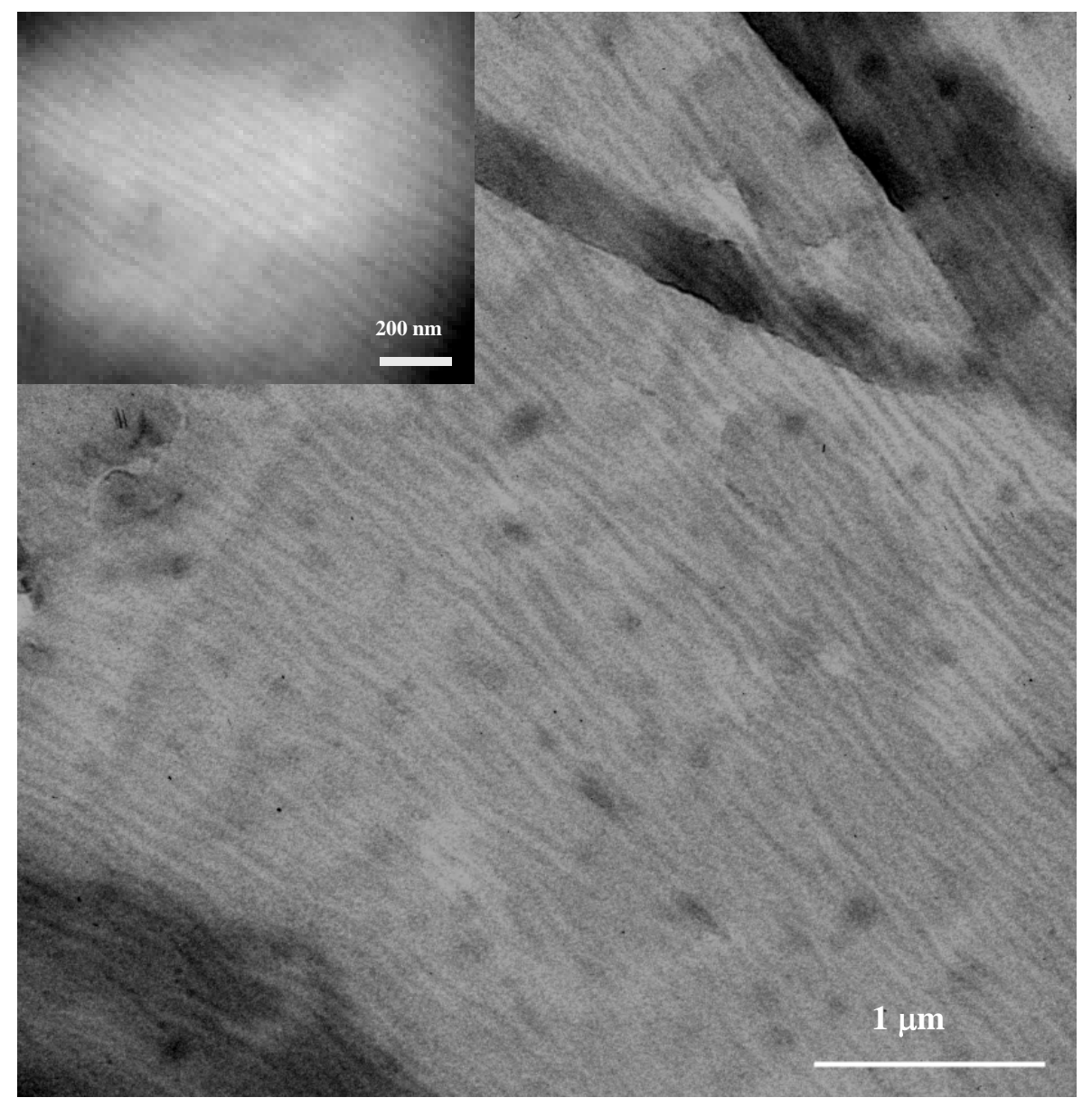

Figure 3S(a). TEM image of PANI(PEOPA) nanowires in through view. The inset image shows the nanowires at higher magnification. 


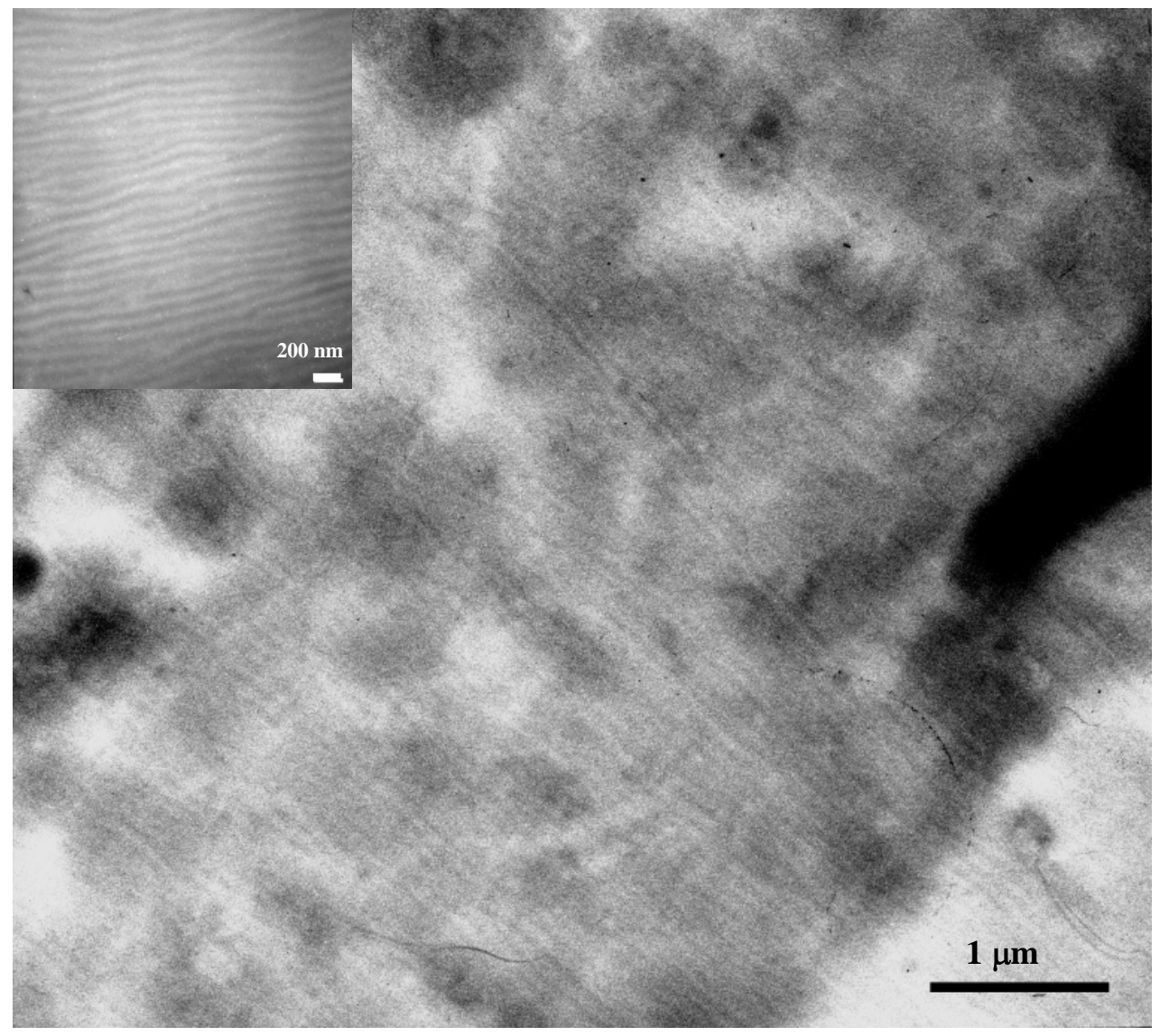

Figure 3S(b). TEM image of PANI(PEOPA) nanowires in edge view. The inset image shows the nanowires at higher magnification. 


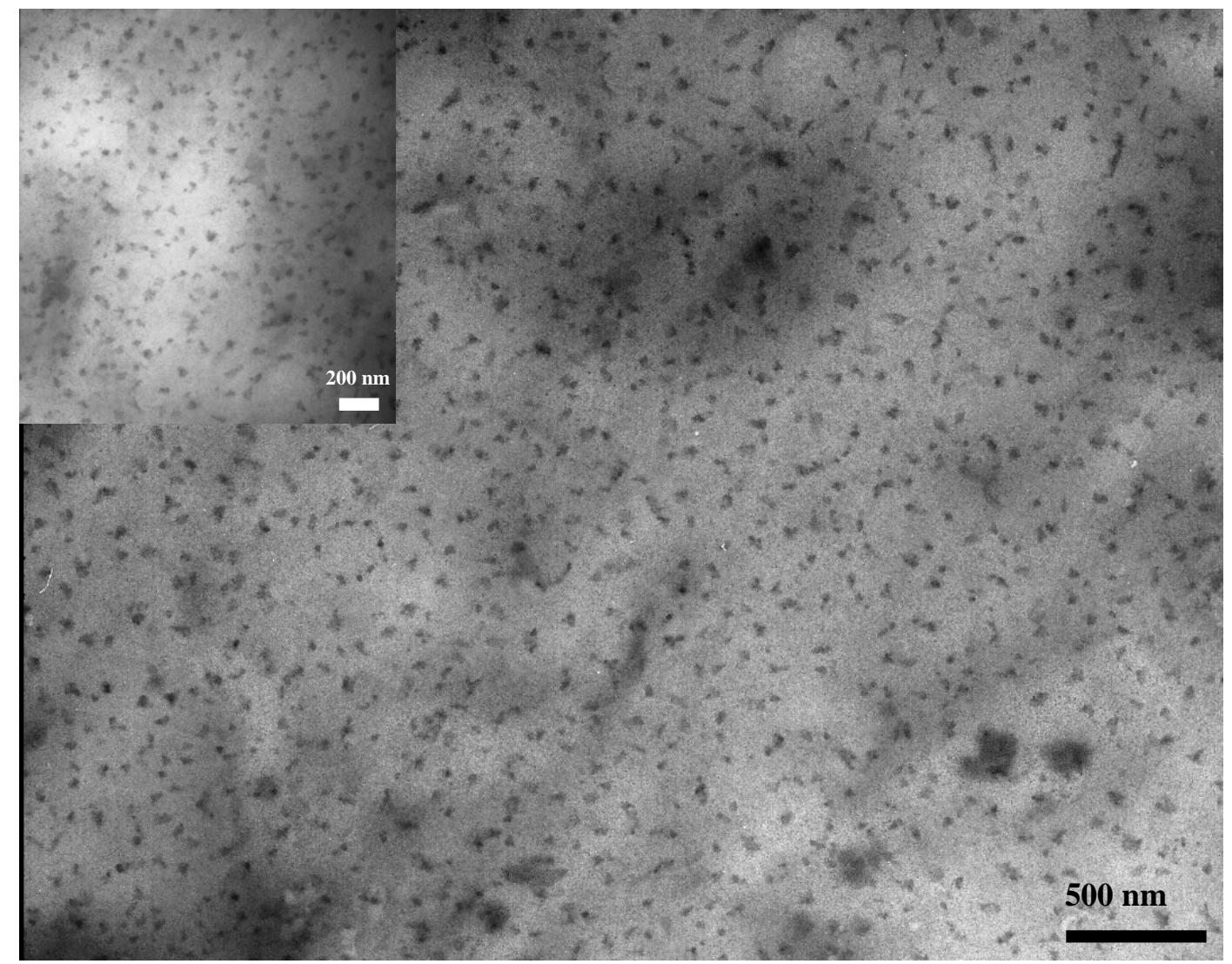

Figure 3S(c). TEM image of PANI(PEOPA) nanowires in end view. The inset image shows the nanowires end at higher magnification. 

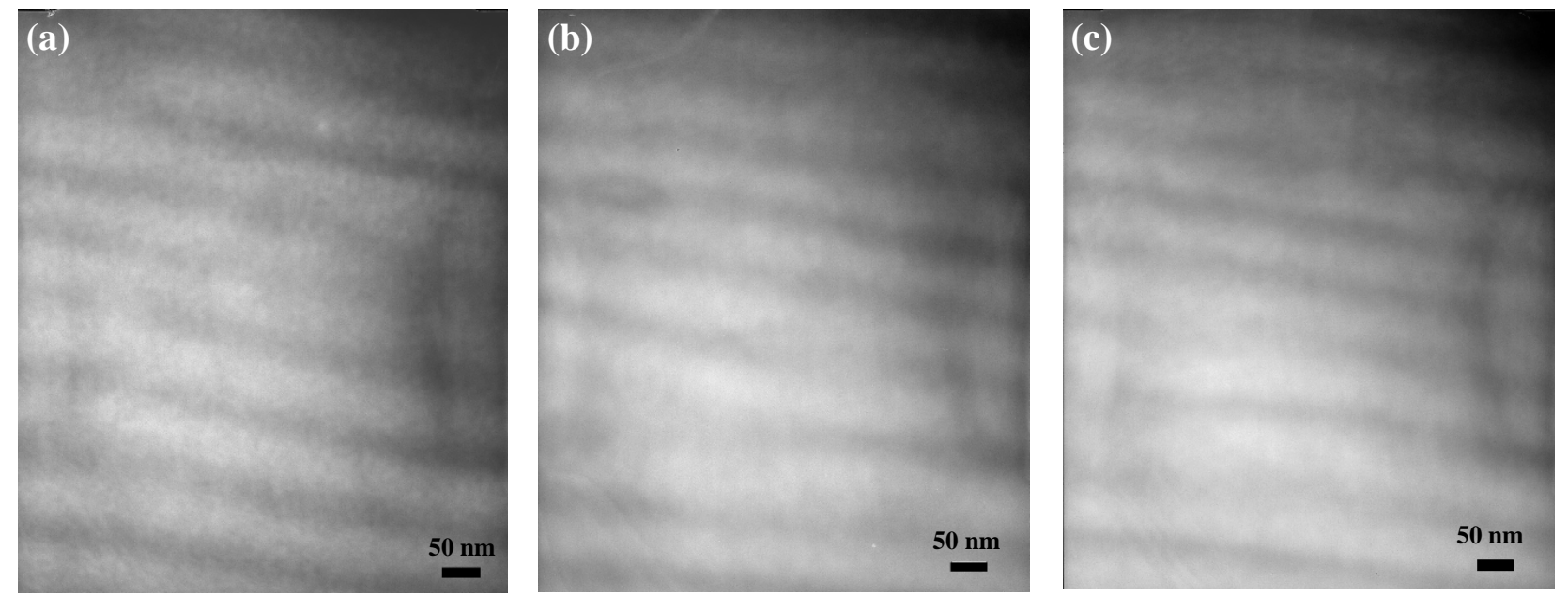

Figure 4S. TEM images obtained from PANI(PEOPA) complex in edge-view: (a) sample tilted $20^{\circ}$ in the anti-clockwise direction, (b) no tilting of the sample, (c) sample tilted $20^{\circ}$ in the clockwise direction. TEM images obtained by tilting showed that the thickness of the layers and contrast in the images remains unaltered. This unambiguously shows that the nanostructures observed from TEM are rod in shape. 
Brief result showing the formation of oriented nanowires in the blend of poly(2-phenyl-3-phenyl-4-(3',7'-dimethyloctyloxy)-1,4-phenylene vinylene) (DPO-PPV) with 4-n-dodecylresorcinol (DR)

The poly(2-phenyl-3-phenyl-4-(3',7'-dimethyloctyloxy)-1,4-phenylene vinylene) (DPO-PPV) was synthesized using method reported elsewhere. ${ }^{1}$ The number average molecular weight $\left(\mathrm{M}_{\mathrm{n}}\right)$ and the polydispersity index of the DPO-PPV was $415,196 \mathrm{~g} / \mathrm{mol}$ and 1.107 , respectively. 4-ndodecylresorcinol (DR) was acquired from Tokio Kasei. The DPO-PPV(DR $)_{x}$ complexes were prepared by mixing the two components in toluene followed by removal of the solvent in vacuo. Here " $x$ " denoted the molar ratio of DR to the monomer unit of DPO-PPV.

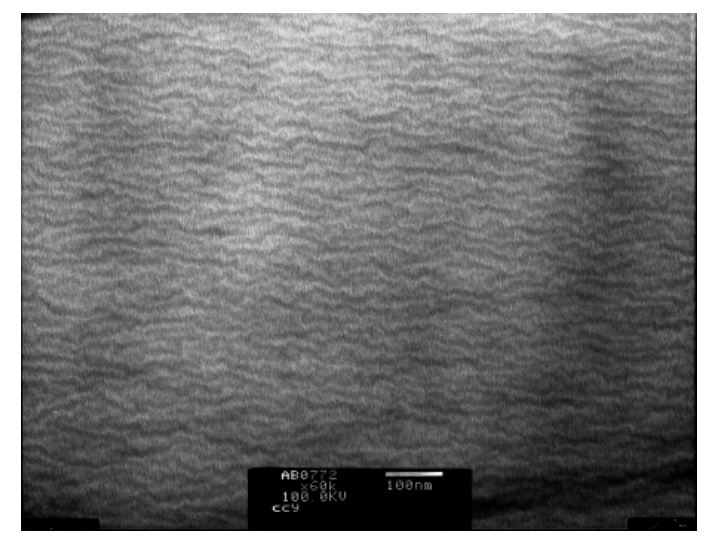

Figure 5S. TEM micrograph showing the nanowire-like morphology for DPO-PPV $(\mathrm{DR})_{\mathrm{X}}$ complex with $\mathrm{x}$ = 1.0. The dark region corresponds to the DPO-PPV-rich domain due to preferential staining by $\mathrm{OsO}_{4}$ while the bright regions consists of the crystalline DR.

\section{References}

1. Lin, K.-F. J. Polym. Sci. Part A: Polym. Chem. 2003, 41, 2180, 